\title{
Field Implementation of a Multi-Modal Prophylactic Strategy (Dinotefuran-Permethrin-Pyriproxyfen and Milbemycine Oxime-Praziquantel) Against Canine Vector-Borne Diseases in Corsica
}

\author{
Younes Laidoudi ${ }^{1,2}$, Hacène Medkour ${ }^{1,2}$, Djamel Tahir ${ }^{1,2}$, Handi Dahmana ${ }^{1,2}$, Jean-Lou Marié ${ }^{3}$, Marie \\ Varloud $^{4}$, Oleg Mediannikov ${ }^{1,2}$ and Bernard Davoust ${ }^{1,2,3^{*}}$ \\ ${ }^{1}$ Aix Marseille Univ, IRD, AP-HM, MEPHI, IHU Méditerranée Infection, 19-21, Bd Jean Moulin, 13385 Marseille \\ Cedex 05, France; younes.laidoudi@yahoo.com (Y.L.); hacenevet1990@yahoo.fr (H. M.); handy92@hotmail.fr \\ (H.D.); olegusss1@gmail.com (O. M.); bernard.davoust@gmail.com (B. D.) \\ 2IHU Méditerranée Infection, 19-21, Bd Jean Moulin, 13385 Marseille Cedex 05, France; djamel.tahir@yahoo.fr \\ (D.T.); handy92@hotmail.fr (H.D.) \\ ${ }^{3}$ Animal Epidemiology Working Group of the Military Health Service, Marseille, France; jean- \\ lou.marie@intradef.gouv.fr (J.L.M.) \\ ${ }^{4}$ Ceva Santé Animale, 10, Av de la Ballastière, 33500 Libourne, France; marie.varloud@ceva.com (M.V.) \\ Correspondence: bernard.davoust@gmail.com; Tel.: +33-0413732401.
}

\section{Running title: Prevention against canine vector-borne diseases in Corsica}

\begin{abstract}
With a mild Mediterranean climate, Corsica is endemic with canine vector-borne diseases (CVBDs) such as dirofilariosis, leishmaniosis and ehrlichiosis. The aim of the present study was to evaluate a monthly multi-modal prophylactic (MMP) strategy against CVBDs occurring on the island. The study was conducted as a comparative field trial in which eighty dogs were allocated into two groups: (i) a test group consisted of 25 dogs under the MMP [per-os administration of 1.5 tablet of milbemycine oxime-praziquantel and a topical line-on application of a $3.6 \mathrm{~mL}$ solution of dinotefuranpermethrin-pyriproxyfen] and (ii) a control group under various real-life prophylactic treatments (RLP) based on the use of ectoparasiticide products [different formulations: deltamethrin, fluralaner, fipronil] and/or macrocyclic lactone based-products [milbemycin oxime/praziquantel, milbemycin oxime, moxidectin] during the period ranging from June to October 2017. All animals were followed for one year and had blood drawn at day 0, with follow-ups at 6 and 12 months. Samples were screened for filariosis using molecular tools, as well as for leishmaniosis and ehrlichiosis, using indirect immunofluorescence assay (IFA). At the end of the study, no new cases of CVBDs were recorded within the test group. In the control group, the cumulative incidence of CVBDs was $20.0 \%(n=11 ; p=$ 0.015), including dirofilariosis due to Dirofilaria immitis and/or D. repens, with $16.4 \%$ ( $n=9 ; p=0.027)$. Ehrlichiosis was $5.5 \%(n=3 ; p=0.241)$. No new cases of leishmaniosis were detected in the control group. The data illustrated that, unlike the RLP treatment which failed to protect at least $20 \%$ of dogs, the MMP based on the concurrent administration of milbemycine oxime-praziquantel and dinotefuranpermethrin-pyriproxyfen is efficient in protecting dogs against CVBDs in a high-risk area.
\end{abstract}

Keywords: canine vector-borne diseases; mosquito-borne helminths; dirofilariosis; ehrlichiosis; leishmaniosis; dogs; multi-modal prophylaxis; Corsica 


\section{Introduction}

Canine vector-borne diseases (CVBDs) include a group of rapidly spreading and globally distributed infections that are caused by viruses, bacteria, protozoa and helminths [1]. Their transmission requires hematophagous arthropods such as mosquitoes, ticks, fleas, sandflies, tsetse flies, triatomine bugs, blackflies, mites or lice [1]. The geographical expansion and spread of CVBDs is directly related to the climate changes, vector propagation, human and animal travels across the world as well as the lack of field chemoprophylaxis and prevention in the exposed area [2]. The Mediterranean region is known for the emergence, spread and endemicity of several vector-borne diseases. Among CVBD-causing pathogens, Dirofilaria immitis and Dirofilaria repens are the most relevant mosquito-borne helminths $(\mathrm{MBH})$ that threaten humans and pets in the Mediterranean basin. D. immitis induces the heartworm disease (Syn. cardiopulmonary dirofilariosis) in dogs and the pulmonary dirofilariosis in humans, while $D$. repens causes respectively subcutaneous and subcutaneous/ocular dirofilariosis in canine and human hosts [3]. Ehrlichia canis, an obligatory intracellular bacterium transmitted by ticks, is responsible for both canine and human monocytic ehrlichiosis [1]. Leishmania infantum, a sandflyborne protozoan causes canine leishmaniosis and human visceral leishmaniosis, both are often responsible for considerable morbidity if left untreated [4].

Corsica is endemic for a wide range of CVBDs. The prevalence rates in dogs were recorded at $21.3 \%$ [5], for D. immitis. The presence of canine ehrlichiosis was confirmed on the island [6,7]. The cumulative incidence of canine leishmaniosis throughout the past 20 years (from 1993 to 2012) was reported at 42.5\% [8]. Furthermore, several studies reported that Corsica is an epidemiological focus from which the main cases of human and canine vector-borne diseases are imported, such as human leishmaniosis by tourists [9], D. immitis in imported dogs from Corsica $[10,11]$ and human dirofilariosis due to $D$. repens in visitors to the island [12]. Consequently, CVBDs in Corsica need to be further investigated and cost-effective control programs need to be put in place.

Recently, a multi-modal prophylactic (MMP) strategy targeting simultaneously the pathogens and their vectors provided promising results against heartworm. This MMP was implemented by combining the administration to dogs of a macrocyclic lactone with larvicidal activity (Interceptor ${ }^{\circledR}$, Elanco, Neuilly-sur-Seine, France) and a repellent insecticide active against mosquitoes (dinotefuran, pyriproxyfen and permethrin (DPP), Vectra ${ }^{\circledR}$ 3D, Ceva Santé Animale, Libourne, France) $[13,14]$. The MMP were more effective $(100 \%)$ than topical Vectra ${ }^{\circledR} 3 \mathrm{D}(96 \%)$ or Interceptor ${ }^{\circledR}(58 \%)$ in preventing the lactone macrocyclic-resistant strain of $D$. immitis under experimental conditions $[13,14]$.

In addition, DPP was designed to treat and prevent a wide range of external parasitic infestations (ticks, mosquitoes, sandflies, stable flies, fleas and triatomes) [15-17]. However, no data were available on the effectiveness of the MMP strategy against CVBDs in the field. In this field study, we tested the MMP protocol based on the concurrent treatment with a broad-spectrum insecticides (DPP) and a larvicidal products (milbemycine oxime-praziquantel) against the major CVBDs occurring in Corsica, an area well known for its endemicity [1,6-8].

\section{Methods}

\subsection{Study Area}

The study was carried out in Ventiseri $\left(41^{\circ} 55^{\prime} 52.5^{\prime \prime} \mathrm{N}\right.$; 9 $\left.{ }^{\circ} 23^{\prime} 58.3^{\prime \prime} \mathrm{E}\right)$, Solaro Marine $\left(41^{\circ} 50.1114^{\prime} \mathrm{N}\right.$; $\left.9^{\circ} 22.482^{\prime} \mathrm{E}\right)$ and Aleria $\left(42^{\circ} 6^{\prime} 15.293^{\prime \prime N}\right.$; $\left.9^{\circ} 30^{\prime} 44.744 " \mathrm{E}\right)$ on the island. Corsica is some $400 \mathrm{~km}$ from mainland France in the Mediterranean Sea between the European and the African continents. The island is inhabited by some 320,000 people and is visited by more than three million tourists a year [18]. The ecological conditions are characterized by a forest environment (more than $46 \%$ of the total area of the island) and a clement temperature (annual mean of $17^{\circ} \mathrm{C}$ ) with a moderate precipitation $(600-800$ millimeters per annum), which offers a suitable environment for the development, spread and expansion of vector-borne diseases [18,19].

\subsection{Animal Enrollment}


From March 2017 to April 2018, a comparative field trial was carried out. The study was conducted according to the guidelines on data requirements for veterinary medicinal products for the prevention of transmission of vector-borne diseases in dogs and cats [20]. Dogs were enrolled and analyzed according to the following criteria: (i) the consent of dog owners was confirmed at the enrollment at Day (D) 0. (ii) The dogs were negative for at least one of the selected CVBDs, (iii) dogs did not present clinical signs compatible with heartworm, canine leishmaniosis or ehrlichiosis threatening the dog's life during the follow-up, (iii) the dogs were at least one year old at the time of registration and were receiving regular prophylactic treatment based on ectoparasiticide and/or macrocyclic lactone basedproducts. However, the exclusion criteria were: animals not remaining permanently in their residence throughout the study period or refusal by owners to participate. The trial evaluation was based on the incidence of CVBDs at the middle and at the end of the trial. Once the laboratory diagnosis had been established after each visit, the results of the screening were communicated to the owners of the animals or their veterinarians in order to implement the appropriate treatment; the preventive treatment was maintained for these dogs, and they were not excluded from the study.

\subsection{Canine Population, Treatment Protocol and Blood Collection}

In March 2017 (D0), 114 dogs were enrolled and allocated into two groups:

i) The test group (test treatment group, $\mathrm{n}=36$ ) consisted of military working dogs (MWD) housed individually in wire cages in a kennel located in Ventiseri (Figure 1a). Dogs received monthly multi-modal prophylactic treatment (MMP) [per os administration of 1.5 tablet of milbemycine oxime-praziquantel (Milbactor ${ }^{\circledR}$, Ceva Santé Animale, Libourne, France) and a topical line-on application of a $3.6 \mathrm{~mL}$ solution of dinotefuran-permethrin-pyriproxyfen (Vectra ${ }^{\circledR}$ D, Ceva Santé Animale)].

ii) The control group (active control, $\mathrm{n}=78$ ) consisted of civilian dogs of Solaro Marine and those from the kennel of Aleria. All dogs had an outdoor lifestyle in wire cages or free in gardens (Figure 1b). These dogs received various real-life prophylactic treatments (RLP).
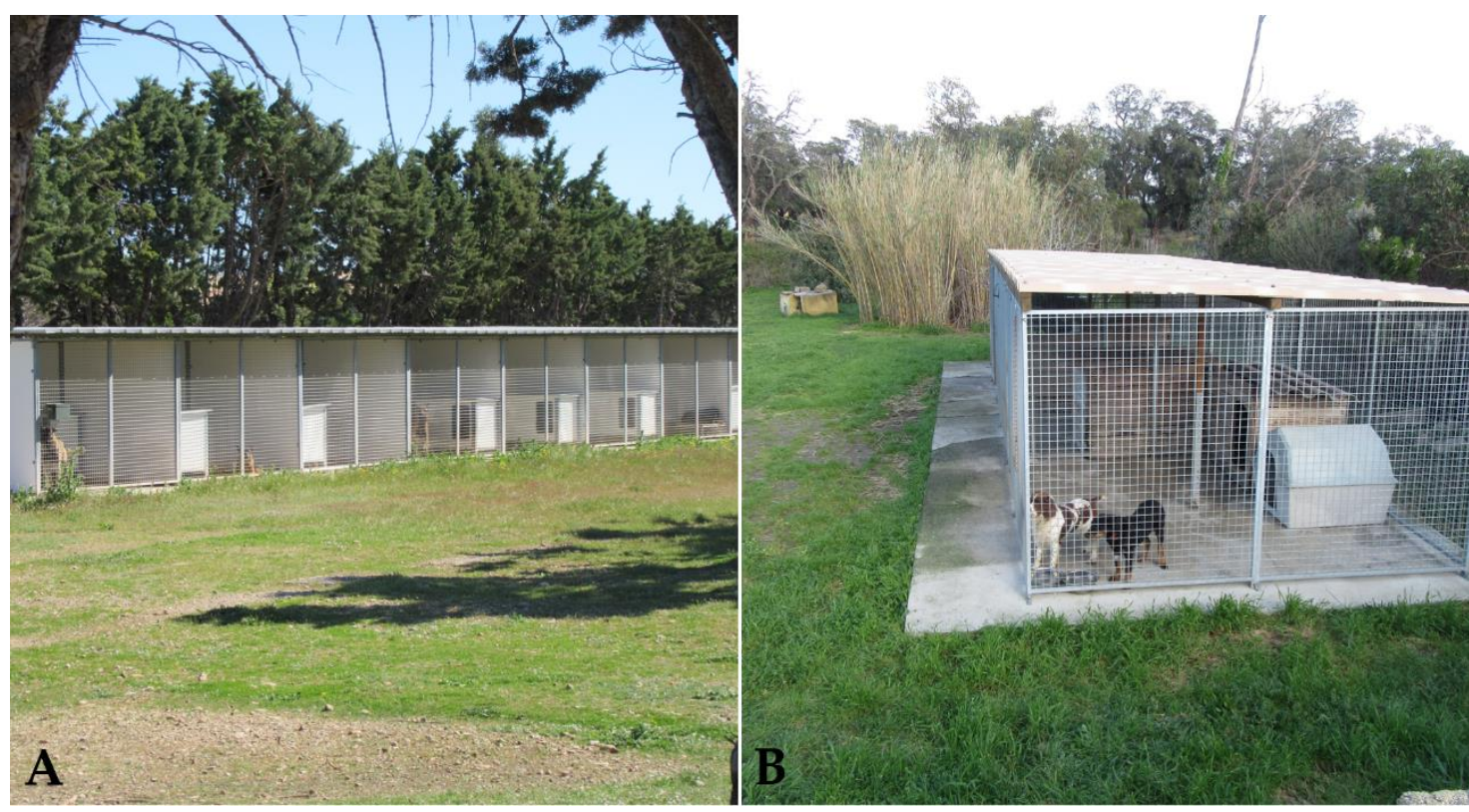

Figure 1. Photo shoot showing house conditions of (A) military working dogs and (B) civilian dogs.

Three and ten dogs were lost for follow-up at D0 +6 months, 8 and 13 other dogs were lost for follow-up at D0+ 12 months from the first and the second group, respectively (Figure 2). At the end of the study, a cohort of 80 dogs was reconstituted for a one-year follow-up, consisting of 25 MWD (test 
group), represented by 23 males and 2 females, aged 2 to 11 years old, with a median of 4 years, and 55 civilian dogs (control group), consisting of 31 males and 24 females aged from 1 to 17 years old, with a median age of 5 years. The 55 civilian dogs included 5 sheepdogs, 25 hunting dogs and 25 pets. Blood on citrate and plain tubes for serum harvesting were collected by a veterinarian from the dogs' cephalic veins. Each dog was sampled and tested 3 times at 6-month intervals (D0, D0+6 and D0+12 months) for the presence of heartworm (D. immitis and its endosymbiotic Wolbachia), D. repens, Acanthocheilonema reconditum, L. infantum and E. canis infections.

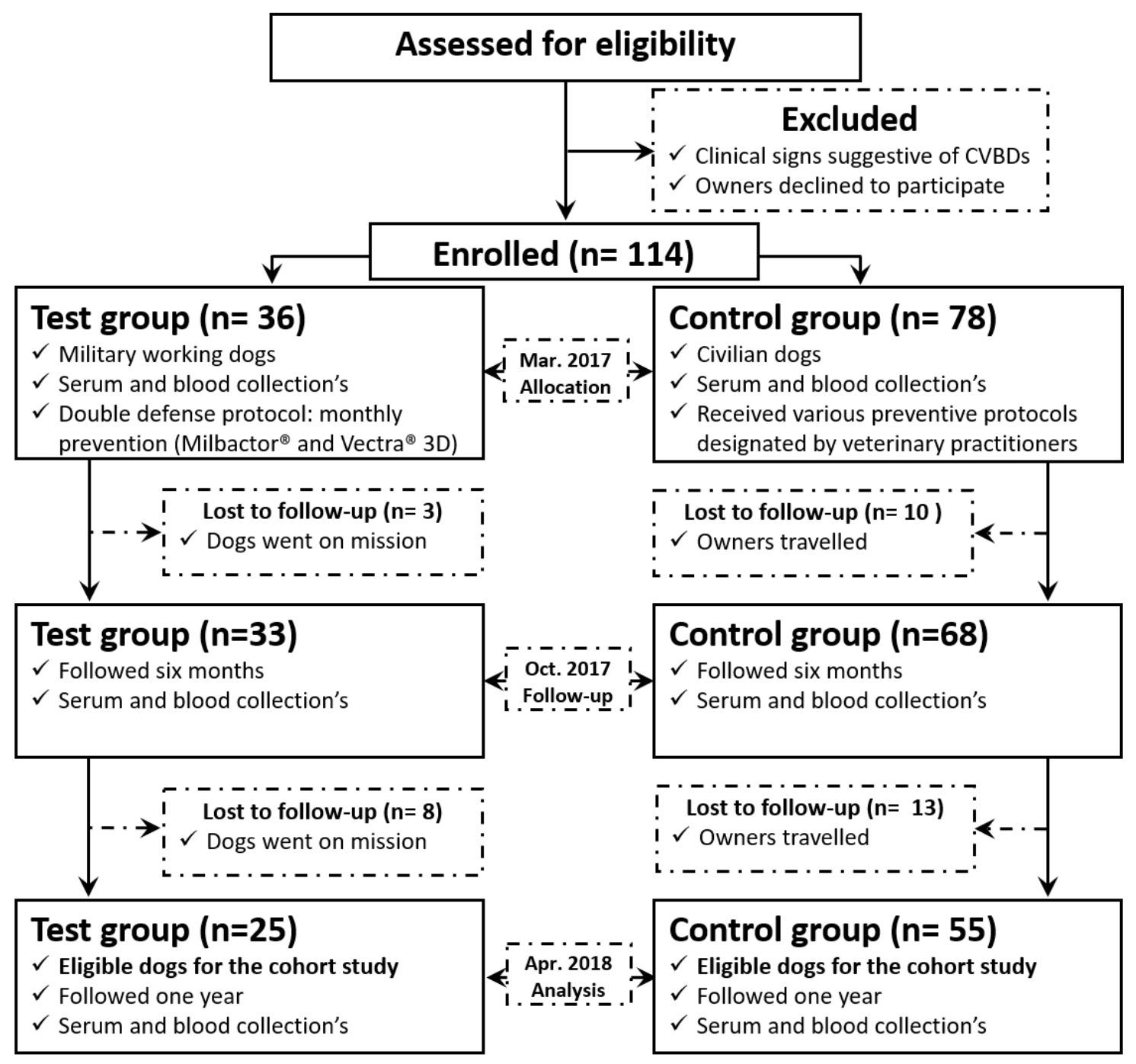

Figure 2. Flow diagram showing the semi-annual evolution of the cohort.

\subsection{Laboratory Analysis}

\subsubsection{Molecular Detection of Filarial Infections}

Genomic DNA was extracted from the citrate blood using the Biorobot EZ1 System with the EZ1 DNA tissue kit (Qiagen, Courtaboeuf, France) according to the manufacturer's instructions. Dogs were screened at each examination for the presence of $D$. immitis, D. repens and A. reconditum DNA using a triplex qPCR assay (Triplex TaqMan COI qPCR) [21]. This molecular assay is able to detect up to $5 \times 10^{-}$ ${ }^{1}$ microfilaria per milliliter of blood (corresponding to $2.5 \times 10^{-3} \mathrm{mfs}$ per qPCR reaction) of each species simultaneously. A second qPCR (ftsZ-WD.immitis- qPCR) targeting the specific Wolbachia endosymbiont of $D$. immitis was used to complete the molecular diagnosis of heartworm infection [22]. The detection of the specific genotype of Wolbachia in filarial infections improved the diagnosis value 
and resolved the occult infections [21][22], as the endosymbiont are released by the filarial worms irrespective of their gender [23,24]. The detection of Wolbachia DNA together with that of its filarial host (e.g. D. immitis) had 100 and $99.3 \%$ of sensitivity and specificity respectively [21].

qPCRs reactions were carried out in total volume of $20 \mu \mathrm{L}$, containing $5 \mu \mathrm{L}$ of DNA template, 10 $\mu \mathrm{L}$ of Master Mix Roche (Eurogentec), $0.75 \mu \mathrm{L}$ of each primer (20 $\mu \mathrm{M}$ concentration) per reaction for the triplex and $0.5 \mu \mathrm{L}$ for simplex qPCR, $0.5 \mu \mathrm{L}$ of both UDG and each probe ( $5 \mu \mathrm{M}$ concentration). Finally, the volume was made up to $20 \mu \mathrm{L}$ using ultra-purified water free of DNAse-RNAse, i.e. $1.5 \mu \mathrm{L}$ for the triplex and $3 \mu \mathrm{L}$ for the simplex qPCR. The qPCR run protocol included two hold steps at $50^{\circ} \mathrm{C}$ for 2 minutes, followed by a cycle at $95^{\circ} \mathrm{C}$ for 15 minutes, and 40 cycles of two steps each $\left(95^{\circ} \mathrm{C}\right.$ for $30 \mathrm{~s}$ and $60^{\circ} \mathrm{C}$ for $30 \mathrm{~s}$ ). These reactions were performed in a thermal cycler CFX96 Touch detection system (BioRad, Marnes-la-Coquette, France) after activating the readers of the dyes used in each qPCR system.

\subsubsection{Serological Analysis}

Sera were individually screened for canine leishmaniosis and monocytic ehrlichiosis antibodies using the quantitative indirect immunofluorescence test. The assay was performed using L. infantum and E. canis commercialized antigens (Zoetis, Lyon, France) according to the manufacturer's instructions. A two-fold dilutions (1:50 and 1:100) of sera (including positive and negative sera) were prepared in phosphate buffered saline (PBS), and a dilution of 1:100 was selected as the cut-off value. Slide wells were sensitized by $20 \mu \mathrm{L}$ or $10 \mu \mathrm{L}$ of L. infantum or E. canis commercial antigens (Zoetis) respectively. Later, $20 \mu \mathrm{L}$ of each serum dilution was applied per well and slides were incubated for 30 $\min$ at $37^{\circ} \mathrm{C}$. Sensitized slides were washed twice with PBS for 5 minutes and once with distilled water. $20 \mu \mathrm{L}$ of rabbit anti-dog IgG conjugated with fluorescein isothiacyanate (FITC) (Sigma-Aldrich, St Louis, MO, USA) were added into each well. Slides were immediately incubated at $37^{\circ} \mathrm{C}$ for 30 minutes. The washing procedure was repeated as described above, and cover slips were mounted in mounting medium. Slides were examined under a UV light microscope (Leica ${ }^{\circledR}$ DM LB2, Gx40).

\subsection{Incidence Estimation and Data Analysis}

Positive dogs are defined here as dogs positive for one or more selected CVBDs. Chi-Square $\left(x^{2}\right)$ and exact Fisher $(F)$ tests were applied to compare the proportion of infected dogs in test and control groups at Day 0 (baseline). The incidence was first determined using the incidence density rate (IDR) represented by the rate of positive dogs (i.e., new infections) observed at every 6th month of follow-up for each pathogen or group of pathogens. IDRs were calculated for each CVBD every 6 months over the follow-up (i.e., considering only results of Day +6 and Day +12 months in comparison to Day 0 and Day +6 months respectively). In addition, the cumulative incidence (CI) rates [25] for each pathogen or group of pathogens were estimated from the half-year-crude incidence (i.e., considering only results of the new infections after the sixth and twelfth month, regardless of what happened in between). Finally, Gray's test [26] was applied to compare cumulative incidence rates in test and control groups. Significance was defined at alpha $\leq 0.05$. Statistical analysis was performed using Addinsoft. 2018. XLSTAT 2018: Data Analysis and Statistical Solution for Microsoft Excel. Paris, France (2018). The protection level of MMP was expressed in terms of the percentage of success in blocking the transmission of the pathogen or group of pathogens, calculated at the end of the follow-up using the following formula: Protection $(\%)=100 \times[(\mathrm{CIt}-\mathrm{CIc}) / \mathrm{CIc}]$, where the CIt and CIc represent the cumulative incidence rate of CVBDs in test and control group respectively [20].

\section{Results}

At Day 0 (baseline), the infectious status of test and control groups were homogeneous $(p>0.05)$ in terms of dogs positive for pathogens or groups of pathogens [i.e., canine vector-borne diseases (CVBDs) and mosquito-borne helminths (MBHs)]. Prior to the trial, 20 and 21.8\% of dogs were positive for at least one of the selected CVBD, in test and control group respectively (Table 1). Only E. canis was 
detected in five dogs (20\%) from the test group, while heartworm, L. infantum and E. canis were respectively detected in $4(7.2 \%), 5(9.1 \%)$ and $4(7.2 \%)$ dogs in the control group (Table 1$)$. All dogs were negative for $D$. repens at the enrollment.

Table 1. Number and prevalence of infections by pathogens or groups of pathogens [i.e., canine vector-borne diseases (CVBDs) and mosquito-borne helminths (MBHs)] detected at baseline (March 2017).

\begin{tabular}{cccc}
\hline \multirow{2}{*}{ Pathogens } & \multicolumn{2}{c}{ Positive dogs (\%) } & \multirow{2}{*}{ Significance ( $\boldsymbol{p}$-value) } \\
\cline { 2 - 3 } & Test group (N=25) & Control group (N=55) & \\
\hline CVBDs & $5(20)$ & $12(21.8)$ & 0.85 \\
\hline MBHs & $0(0)$ & $4(7.2)$ & 0.304 \\
\hline Wolbachia of $D$. immitis & $0(0)$ & $3(5.4)$ & 0.548 \\
D. immitis & $0(0)$ & $3(5.4)$ & 0.548 \\
Heartworm (combined detection) & $0(0)$ & $4(7.2)$ & 0.304 \\
\hline D. repens & $0(0)$ & $0(0)$ & $/ /$ \\
\hline L. infantum & $0(0)$ & $5(9.1)$ & 0.317 \\
\hline E. canis & $5(20 \%)$ & $4(7.2)$ & 0.129 \\
\hline
\end{tabular}

At the end of the follow-up, 80 dogs ( 25 from the test group and 55 from the control group) were included in the cohort study. Unlike the test group, dogs from the control group were vulnerable to at least one CVBD irrespective of their treatment. However, dogs receiving the concurrent treatment with a repellent insecticide and macrocyclic lactone-based products remained the most protected (Table 2).

No new cases of CVBDs were recorded within the test group under the MMP which corresponds to a protection of $100 \%$. However, $2(3.6 \%)$ and $9(16.4 \%)$ new cases of CVBDs were recorded in control group at 6 and 12 months of follow-up respectively. The incidence density rates and the cumulative incidences of CVBDs are detailed in Table 3. The highest IDR was observed for MBHs at Day +12 months in control group, wherein $14.6 \%(\mathrm{n}=8)$ and $1.8 \%(\mathrm{n}=1)$ of dogs were positive for heartworm and D. repens respectively. No new cases of $L$. infantum were reported in either group throughout the follow-up. The IDRs of ehrlichiosis were $3.6 \%(\mathrm{n}=2)$ and $1.8 \%(\mathrm{n}=1)$ at Day +6 months and Day +12 months respectively, in the control group. The CI for all CVBDs, grouped MBHs and D. immitis were significantly higher in the control than in the test group. Finally, no difference was observed between the groups for D. repens, L. infantum and E. canis. 
Table 2. Treatment and cumulative number of newly infected dogs in terms of CVBDs-causing pathogens or groups of pathogens [i.e., canine vector-borne diseases (CVBDs) and mosquito-borne helminths (MBHs)] detected by serology or qPCR in dogs at Day 0, 6 and 12-months of follow-up.

\begin{tabular}{|c|c|c|c|c|c|c|c|c|c|}
\hline Group & Treatment & Treatment period & $\operatorname{Dogs}(\mathrm{N})$ & $\begin{array}{l}\text { D. immitis } \\
\text { and/or W.Dimm }\end{array}$ & D. repens & L. infantum & E. canis & MBHs & CVBDs \\
\hline $\begin{array}{l}\text { Test } \\
\text { group }\end{array}$ & $\begin{array}{l}\text { a. Milbemycine oxime/praziquantel (Milbactor®) } \\
\text { b. Dinotefuran/Permethrin/Pyriproxyfen (Vectra }{ }^{\circledR} \text { 3D) }\end{array}$ & Once a month throughout the year* & 25 & 0 & 0 & 0 & 0 & 0 & 0 \\
\hline \multirow{9}{*}{$\begin{array}{l}\text { Control } \\
\text { group } \\
\text { (RLP) }\end{array}$} & $\begin{array}{l}\text { a. Fipronil (Flivox®) } \\
\text { b. Milbemycin oxime/praziquantel (Milbemax®) } \\
\text { c. Imidacloprid/permethrin (Advantix®) }\end{array}$ & $\begin{array}{l}a \mathrm{a} \text { and b: Once a month from June to } \\
\text { October* } \\
\text { c: Two times per year (March and } \\
\text { October) }\end{array}$ & 38 & 1 & 1 & $1^{* *}$ & 1 & 2 & 3 \\
\hline & Imidacloprid and permethrin (Advantix $®$ ) & $\begin{array}{l}\text { Once every two months from June to } \\
\text { September }\end{array}$ & 2 & 1 & 0 & 0 & 0 & 1 & 1 \\
\hline & $\begin{array}{l}\text { a. Fluralaner (Bravecto®) } \\
\text { b. Milbemycin oxime/praziquantel (Milpro®) }\end{array}$ & Once every 6 months* & 2 & 0 & 0 & 0 & 1 & 0 & 1 \\
\hline & Deltamethrin (Scalibor@) & Once every 6 months & 3 & 2 & 0 & 0 & 0 & 2 & 2 \\
\hline & Fipronil (Frontline®) & Occasionally in summer & 2 & 1 & 0 & 0 & 0 & 1 & 1 \\
\hline & Moxidectin (Guardian $®$ ) & Occasionally in summer & 3 & 1 & 0 & 0 & 0 & 1 & 1 \\
\hline & Milbemycin oxime (Interceptor $\left.{ }^{\circledR}\right)$ & $\begin{array}{l}\text { Once per year (between June to } \\
\text { September) }\end{array}$ & 3 & 1 & 0 & 0 & 1 & 1 & $1^{*}$ \\
\hline & Milbemycin oxime/praziquantel (Milbemax®) & Once every 6 months & 2 & 1 & 0 & 0 & 0 & 1 & 1 \\
\hline & Total & // & 55 & 8 & 1 & 0 & 3 & 9 & 11 \\
\hline
\end{tabular}

* Samples positive for at least 2 CVBDs, which were considered only once in the calculation of grouped CVBDs.

W.Dimm: Wolbachia endosymbiont of D. immitis.

RLP: real-life prophylactic treatments. 
Table 3. Incidence density rates (IDR) and cumulative incidences (CI) of canine vector-borne diseases (CVBDs), mosquito-borne helminths (MBHs) or for each single pathogen. $P$-values compare the $\mathrm{CI}$ rates in both test and control groups.

\begin{tabular}{|c|c|c|c|c|c|c|}
\hline \multirow[b]{2}{*}{ Pathogens/time } & \multicolumn{2}{|c|}{ IDRs (\%) } & \multicolumn{2}{|c|}{ Cumulative incidences (\%) } & \multicolumn{2}{|c|}{$\begin{array}{c}\text { Pairwise comparison } \\
\text { (Gray's test) }\end{array}$} \\
\hline & Test group & Control group & Test group & Control group & Statistic & $p$-value \\
\hline \multicolumn{7}{|c|}{ CVBDs (positive for at least one CVBD-causing pathogens) } \\
\hline Day +6 months & 0 & 3.6 & \multirow{2}{*}{0} & \multirow{2}{*}{20} & \multirow{2}{*}{6.06} & \multirow{2}{*}{0.014} \\
\hline Day +12 months & 0 & 16.4 & & & & \\
\hline \multicolumn{7}{|c|}{ MBHs (dirofilariosis) } \\
\hline Day +6 months & 0 & 0 & \multirow{2}{*}{0} & \multirow{2}{*}{16.4} & \multirow{2}{*}{4.55} & \multirow{2}{*}{0.033} \\
\hline Day +12 months & 0 & 16.4 & & & & \\
\hline \multicolumn{7}{|c|}{ Heartworm (combined detection of D. immitis and its endosymbiotic Wolbachia) } \\
\hline Day +6 months & 0 & 0 & \multirow{2}{*}{0} & \multirow{2}{*}{14.6} & \multirow{2}{*}{4.0} & \multirow{2}{*}{0.046} \\
\hline Day +12 months & 0 & 14.6 & & & & \\
\hline \multicolumn{7}{|c|}{ D. repens } \\
\hline Day +6 months & 0 & 0 & \multirow{2}{*}{0} & \multirow{2}{*}{1.8} & \multirow{2}{*}{0.46} & \multirow{2}{*}{0.500} \\
\hline Day +12 months & 0 & 1.8 & & & & \\
\hline \multicolumn{7}{|c|}{ L. infantum } \\
\hline Day +6 months & 0 & 0 & \multirow{2}{*}{0} & \multirow{2}{*}{0} & \multirow{2}{*}{-} & \multirow{2}{*}{-} \\
\hline Day +12 months & 0 & 0 & & & & \\
\hline \multicolumn{7}{|c|}{ E. canis } \\
\hline Day +6 months & 0 & 3.6 & \multirow{2}{*}{0} & \multirow{2}{*}{5.5} & \multirow{2}{*}{1.37} & \multirow{2}{*}{0.241} \\
\hline Day +12 months & 0 & 1.8 & & & & \\
\hline
\end{tabular}




\section{Discussion}

To the best of our knowledge, this is the first reported field study demonstrating the efficacy of the consistent administration of larvicidal (macrocyclic lactones) + vector prevention products (repellents) as a MMP against CVBDs in endemic areas, and the first European field study confirming the year-round efficacy of the MMP on a monthly schedule against CVBDs. The MMP was first designed to protect against vector bites and the development of larval stages in both the resistant and the non-resistant strains of $D$. immitis and should be efficient in blocking the transmission of dirofilariosis despite the exposure of dogs to infected mosquitoes [13]. In the field, Labarthe et al. (2015), demonstrated that the monthly administration of topical moxidectin + imidacloprid to dogs was highly effective (100\%) in preventing the transmission of D. immitis in a high risk area [27].

Because dogs should be shown to be exposed to transmission risk in a given area, we proceeded to demonstrate the effectiveness of preventive treatment in a zone in which the certainty that transmission exists. The percentage of positive tests for grouped pathogens [i.e. D. immitis, D. repens. L. infantum and E. canis] was above $20 \%$ in both groups thereby demonstrating how intense the challenge was and confirming the very high risk of CVBD previously reported in the site study [5-9]. Therefore, the severe life-threatening condition of these infections as well as their zoonotic potential highlights the real need for a cost-effective chemoprophylaxis easy to implement in order to control transmission.

For obvious ethical reasons, the demonstration of MMP efficacy was based on a comparison with real-life preventive strategies (treated with several existing approved products irrespective of the treatment period or the number of dogs in the subgroups) as an active control instead of an untreated control. The active control groups are often used to demonstrate the efficacy of new treatments on the field [28-30].

Our findings indicate that MMP treatment using Milbactor ${ }^{\circledR}$ and Vectra ${ }^{\circledR} 3 \mathrm{D}$ is efficient under field conditions to block the transmission of single and multiple infections with CVBD caused by $D$. immitis, D. repens, L. infantum and E. canis. A significantly higher level of protection against all these CVBDs was observed in our study, in contrast to the control group, in which dogs were vulnerable to CVBDs ( $n=11, C I=20 \%)$. The protection success in the test group compared to the control group is expected to be the result of different elements. Firstly, the compliance in military working dogs is expected to be higher than in civilian dogs. As far as civilian dogs are concerned, surveys conducted in France among dog owners indicate that the compliance rate of dogs is low, with an average of $6 \%$ [31]. Owners' poor or noncompliance due to incorrect or inconsistent administration of preventive treatments was the main reason for prevention failures [32]. In addition, the synergistic effect due to the simultaneous combination of a macrocyclic lactone and a parasiticide-repellent product provides better protection against CVBDs and in particular against MBHs, with respect to which both products are active. The combination milbemycin oxime/praziquantel, like other macrocyclic lactones, was designed to prevent dirofilarial infections in dogs [33]. DPP has a proven record of effectiveness in preventing a broad spectrum of vectors such as ticks, mosquitoes, sandflies, stable flies, fleas and triatomes [15-17], and this partly explains the successful prevention of the other CVBDs in the present study. Another key success factor is the year-round chemoprophylaxis which appears to be more adapted to the active vectors in the area compared to the 5-month seasonal protection in the control group, despite the use of both a macrocyclic lactone and a repellent insecticide in some of the dogs in that group (Table 2). In southern Europe as well as in Corsica, a year-round active vector such as Stegomyia albopicta (a main vector of Dirofilaria spp.) has recently been identified [5,34]. The yearround chemoprophylaxis against this vector is recommended in Southern Europe [35]. Indeed, the protection level depends on several factors related to epidemiological conditions, host, vectors and the pathogen itself [36]. The European Scientific Counsel Companion Animal Parasites (ESCCAP), the European Society for Dirofilariosis and Angiostrongylosis (ESDA), and the American Heartworm Society (AHS) recommend the preventive strategies combining the administration of macrocyclic lactones with vector prevention products (repellents) [37-38]. This study illustrates the usefulness of 
this strategy to protect dogs from dirofilariosis and from other vector-borne diseases occurring at the same time and throughout the year; chemoprophylaxis should be implemented in high-risk areas such as the Mediterranean region [12].

The treatment of the vector-borne diseases such as heartworm can be life-threatening, expensive and complex [3,39]. Canine leishmaniosis is potentially fatal if left untreated, besides being a major zoonosis. Current treatments for leishmaniosis are very expensive and difficult to implement with limitations such as adverse reactions and relapses [35]. The concept of multimodal prevention can be complemented by other products, such as vaccines against leishmaniosis, which are available in Europe [35] and can improve protection for dogs when combined with vector-borne protection at a lower cost compared to the complicated treatment of visceral leishmaniosis.

\section{Conclusion}

Besides the direct impact upon mosquito-borne helminths, the purposed multimodal prophylactic strategy based on year-round administration of Vectra ${ }^{\circledR}$ D and Milbactor ${ }^{\circledR}$ on a monthly schedule, provides reliable control against other major canine vector-borne diseases (i.e. L. infantum and E. canis) in high-endemic areas. Multi-modal prophylactic treatment should be promoted on a broader scale, especially in and around the endemic areas, such as southern Europe, in order to stop canine vector-borne diseases spreading to dogs and humans.

\section{Abbreviations}

AHS: American Heartworm Society;

CVBDs: canine vector-borne diseases;

ESCCAP: European Scientific Counsel Companion Animal Parasites;

ESDA: European Society for Dirofilariosis and Angiostrongylosis;

IP: intermittent prophylactic treatment;

MMP: multi-modal prophylactic treatment;

MBHs: mosquito-borne helminths.

Acknowledgments: We thank Mahdi Toumert, Massinissa Hachemi, Sandrine Ferrandi, Marie-Hélène Filippi, Mickaël Boni, Laetitia Guidicelli and all the dog owners for their help.

Conflicts of Interest statement: All the authors declare no conflicts of interest related to this article. One author (Marie Varloud) is currently employee of Ceva Santé Animale. However, there were no conflicts of interest that could have biased the work presented in this paper.

Funding: This study was supported by the Institut Hospitalo-Universitaire (IHU) Méditerranée Infection, the National Research Agency under the program « Investissements d'avenir », reference ANR-10-IAHU-03, the Région Provence-Alpes-Côte d'Azur and European funding FEDER PRIMI. This work was also funded by Ceva Santé Animale (supply of pharmaceutical products).

Availability of data and material: The data supporting the conclusions of this article are included within the article.

Authors' contributions: YL, BD, MV, J-LM and OM designed the study. YL, BD, DT, HD and J-LM intervened in the field study. YL and HM performed the laboratory analyzes. YL, BD, OM designed, and YL carried out the data analysis. YL, BD, MV and OM drafted the manuscript. All authors read and approved the final version of the manuscript.

Consent for publication: Not applicable.

Ethics approval and consent to participate: All owners of dogs gave their informed consent to participate in the study. 


\section{References}

1. Otranto, D.; Dantas-Torres, F.; Breitschwerdt, E.B. Managing canine vector-borne diseases of zoonotic concern: part one. Trends Parasitol. 2009, 25(4), 157-163.

2. Simón, F.; González-Miguel, J.; Diosdado, A.; Gómez, P.J.; Morchón, R.; Kartashev, V. The complexity of zoonotic filariasis episystem and its consequences: A multidisciplinary view. Biomed Res. Int. 2017, 6436130.

3. Simón, F.; Siles-Lucas, M.; Morchón, R.; González-Miguel, J.; Mellado, I.; Carretón, E.; Montoya-Alonso, J.A. Human and animal dirofilariasis: The emergence of a zoonotic mosaic. Clin. Microbiol. Rev. 2012, 25(3), 507-544.

4. Velez, R.; Ballart, C.; Domenech, E.; Abras, A.; Fernández-Arévalo, A.; Gómez, S.A.; Tebar, S.; Muñoz, C.; Cairó, J.; Gállego, M. Seroprevalence of canine Leishmania infantum infection in the Mediterranean region and identification of risk factors: The example of North-Eastern and Pyrenean areas of Spain. Prev. Vet. Med. 2019, 162, 67-75.

5. Tahir, D.; Bittar, F.; Barré-Cardi, H.; Sow, D.; Dahmani, M.; Mediannikov, O.; Raoult, D.; Davoust, B.; Parola, P. Molecular survey of Dirofilaria immitis and Dirofilaria repens by new real-time TaqMan ${ }^{\circledR P C R}$ assay in dogs and mosquitoes (Diptera: Culicidae) in Corsica (France). Vet. Parasitol. 2017, 235, 1-7.

6. Parzy, D.; Baudu, P.; Normand, T.; Audonnet, J.; Davoust, B.; Re, D. Ehrlichia canis phylogenetic analysis of the Borgo (Corsica) strain. Clin. Microbiol. Infect. 2009, 15, 76-78.

7. Dahmani, M.; Davoust, B.; Tahir, D.; Raoult, D.; Fenollar, F.; Mediannikov, O. Molecular investigation and phylogeny of Anaplasmataceae species infecting domestic animals and ticks in Corsica, France. Parasit. Vectors 2017, 10(1), 302.

8. Davoust, B.; Roqueplo, C.; Parzy, D.; Watier-grillot, S.; Marié, J. A twenty-year follow-up of canine leishmaniosis in three military kennels in southeastern France. Parasit. Vectors 2013, 6(1), 323.

9. di Muccio, T.; Scalone, A.; Bruno, A.; Marangi, M.; Grande, R.; Armignacco, O.; Gradoni, L.; Gramiccia, M. Epidemiology of imported leishmaniasis in Italy: Implications for a European endemic country. PLoS One 2015, 10(6), e0129418.

10. Zahler, M.; Glaser, B.; Gothe, R. [Imported parasites in dogs: Dirofilaria repens and Dipetalonema reconditum]. Tierarztl. Prax. 1997, 25(4), 388-392.

11. Pantchev, N.; Etzold, M.; Daugschies, A.; Dyachenko, V. Diagnosis of imported canine filarial infections in Germany 2008 - 2010. Parasitol. Res. 2011, 109 Suppl 1, S61-76.

12. Berger, S.M. Infectious Diseases of France; Gideon Informatics, I., Ed.; 2019th ed.; Gideon Informatics, Inc.: Los angeles, California, USA, 2019; ISBN 978-1-4988-2193-3.

13. McCall, J.W.; Varloud, M.; Hodgkins, E.; Mansour, A.; Dicosty, U.; McCall, S.; Carmichael, J.; Carson, B.; Carter, J. Shifting the paradigm in Dirofilaria immitis prevention: Blocking transmission from mosquitoes to dogs using repellents/insecticides and macrocyclic lactone prevention as part of a multimodal approach. Parasit. Vectors 2017, 10(Suppl 2), 525.

14. McCall, J.W.; Hodgkins, E.; Varloud, M.; Mansour, A.; Dicosty, U. Blocking the transmission of heartworm (Dirofilaria immitis) to mosquitoes (Aedes aegypti) by weekly exposure for one month to microfilaremic dogs treated once topically with dinotefuran-permethrinpyriproxyfen. Parasites and Vectors 2017, 10. 
15. European Medicines Agency. Vectra ${ }^{\circledR}$ 3D Product information EPAR 2015. http://www.ema.europa.eu/docs/en_GB/document_libra.

16. Tahir, D.; Davoust, B.; Almeras, L.; Berenger, J.M.; Varloud, M.; Parola, P. Anti-feeding and insecticidal efficacy of a topical administration of dinotefuran-pyriproxyfen-permethrin spoton (Vectra ${ }^{\circledR 3}$ ) on mice against Stegomyia albopicta (= Aedes albopictus). Med. Vet. Entomol. 2017, 31, 351-357.

17. Tahir, D.; Davoust, B.; Varloud, M.; Berenger, J.M.; Raoult, D.; Almeras, L.; Parola, P. Assessment of the anti-feeding and insecticidal effects of the combination of dinotefuran, permethrin and pyriproxyfen (Vectra ${ }^{\circledR} 3 \mathrm{D}$ ) against Triatoma infestans on rats. Med. Vet. Entomol. 2017, 31, 132-139.

18. Grech-Angelini, S.; Stachurski, F.; Lancelot, R.; Boissier, J.; Allienne, J.F.; Marco, S.; Maestrini, O.; Uilenberg, G. Ticks (Acari: Ixodidae) infesting cattle and some other domestic and wild hosts on the French Mediterranean island of Corsica. Parasit. Vectors 2016, 9(1), 582.

19. Toty, C.; Barré, H.; Le Goff, G.; Larget-Thiéry, I.; Rahola, N.; Couret, D.; Fontenille, D. Malaria risk in Corsica, former hot spot of malaria in France. Malar. J. 2010, 9, 231.

20. European Medicines Agency. Guideline on data requirements for veterinary medicinal products for the prevention of transmission of vector- borne diseases in dogs and cats. 2018, $44,1-11 . \quad$ https://www.ema.europa.eu/en/documents/scientific-guideline/draftguideline-data-requirements-veterinary-medicinal-products-prevention-transmissionvector-borne_en.pdf.

21. Laidoudi, Y.; Davoust, B.; Varloud, M.; Niang, E.H.A.; Fenollar, F.; Mediannikov, O. Development of a multiplex qPCR-based approach for the diagnosis of Dirofilaria immitis, D. repens and Acanthocheilonema reconditum. Parasit. Vectors 2020, 13.

22. Medkour, H.; Laidoudi, Y.; Marié, J.L.: Fenollar, F.; Davoust, B.; Mediannikov, O. Molecular investigation of vector-borne pathogens in red foxes (Vulpes vulpes) from southern France. J. Wildl. Dis. 2020, 56(4).

23. Taylor, MJ.; Hoerauf, A. Wolbachia bacteria of filarial nematodes. Parasitol Today. 1999, 15, 437442.

24. Taylor MJ.; Bandi C, Hoerauf A. Wolbachia bacterial endosymbionts of filarial nematodes. Adv Parasitol. 2005, 60, 245-284.

25. Pepe, M.S.; Mori, M. Kaplan-meier, marginal or conditional probability curves in summarizing competing risks failure time data? Stat. Med. 1993, 12(8), 737-751.

26. Dignam, J.J.; Kocherginsky, M.N. Choice and interpretation of statistical tests used when competing risks are present. J. Clin. Oncol. 2008, 26(24), 4027-4034.

27. Labarthe, N.V.; Willi, L.M.V.; Paiva, J.P.; De Miranda, M.G.N.; Zoreck, K.; Mendes De Almeida, F. Chemoprophylaxis of Dirofilaria immitis (Leidy 1856) infection at a high challenge environment. Parasites and Vectors 2015, 8, 6-9.

28. U.S. Department of Health and Human Services, F.; Administration, D. E 10 Choice of Control Group and Related Issues in Clinical Trials. 2009, 2012.

29. Moraes-Da-Silva, M.D.F.C.V.; Mendes-De-Almeida, F.; Abdalla, L.; Merlo, A.; Paiva, J.P.; Labarthe, N.V. Selamectin for the prevention of canine Dirofilaria immitis infection: Field efficacy in client-owned dogs in a high risk area. Parasites and Vectors 2016, 9, 1-5.

30. Six, R. H., Clemence, R. G., Thomas, C. A., et al. Efficacy and safety of selamectin against 
Sarcoptes scabiei on dogs and Otodectes cynotis on dogs and cats presented as veterinary patients. Vet. Parasitol. 2000, 91(3-4), 291-309.

31. Roussel, C.; Drake, J.; Ariza, J.M. French national survey of dog and cat owners on the deworming behaviour and lifestyle of pets associated with the risk of endoparasites. Parasit. Vectors 2019, 12(1), 480.

32. McTier, T.L.; Kryda, K.; Wachowski, M.; Mahabir, S.; Ramsey, D.; Rugg, D.; Mazaleski, M.; Therrien, C.; Adams, E.; Wolff, T.; et al. ProHeart ${ }^{\circledR}$ 12, a moxidectin extended-release injectable formulation for prevention of heartworm (Dirofilaria immitis) disease in dogs in the USA for 12 months. Parasit. Vectors 2019, 12, 369.

33. di Cesare, A. ; Braun, G. ; di Giulio, E. ; et al. Field clinical study evaluating the efficacy and safety of an oral formulation containing milbemycin oxime/praziquantel (Milbemax®, Novartis Animal Health) in the chemoprevention of the zoonotic canine infection by Dirofilaria repens. Parasit Vectors 2014, 7, 347.

34. Otranto, D.; Dantas-Torres, F.; Brianti, E.; Traversa, D.; Petrić, D.; Genchi, C.; Capelli, G. Vector-borne helminths of dogs and humans in Europe. Parasit. Vectors 2013, 6, 16.

35. Dantas-Torres, F.; Otranto, D. Best practices for preventing vector-borne diseases in dogs and humans. Trends Parasitol. 2016, 32, 43-55.

36. Schorderet-Weber, S.; Noack, S.; Selzer, P.M.; Kaminsky, R. Blocking transmission of vectorborne diseases. Int. J. Parasitol. Drugs Drug Resist. 2017, 7(1), 90-109.

37. Genchi, C.; Kramer, L. ESCCAP: European Scientific Counsel Companion Animal Parasites. 2015, 21, 25-54. https://gallery.mailchimp.com/2dfcfcceccf8b9e02ac641a61/files/Parassiti21.pdf.

38. Nelson, C.T.; McCall, J.W.; Jones, S.; Moorhead, A. Current Canine Guidelines for the Prevention, Diagnosis, and Management of Heartworm (Dirofilaria immitis) Infection in Dogs. Am. Hear. Soc. 2018, 1-35.

39. Bowman, D.D.; Atkins, C.E. Heartworm Biology, Treatment, and Control. Vet. Clin. North Am. - Small Anim. Pract. 2009, 39, 1127-1158. 